\title{
Systemic treatment of hepatocellular carcinoma: from sorafenib to combination therapies
}

\author{
Christoph Roderburg ${ }^{\star \star \ddagger, 1}$, Burcin Özdirik ${ }^{\ddagger 1}$, Alexander Wree ${ }^{1}$, Münevver Demir ${ }^{1}$ \& Frank \\ Tacke ${ }^{1}$ \\ ${ }^{1}$ Department of Hepatology \& Gastroenterology, Charité - University Medicine Berlin, Campus Virchow Klinikum \& Charité \\ Campus Mitte, Berlin, 13353, Germany \\ *Author for correspondence: Christoph.roderburg@charite.de \\ $\ddagger$ Authors contributed equally
}

For almost a decade, systemic therapy of advanced hepatocellular carcinoma (HCC) was limited to the tyrosine kinase inhibitor (TKI) sorafenib. Different agents including checkpoint inhibitors, TKIs and antiVEGFR antibodies demonstrated efficacy in treatment. For the first time, the combination of atezolizumab and bevacizumab, a first-line treatment that is superior to the current standard was identified, potentially changing the way we treat HCC. In this review, we summarize current data on systemic treatment of patients with advanced HCC, focusing on combination therapies comprising immune checkpoint inhibitors, TKIs and locoregional therapies. We elucidate findings from recent trials and discuss such challenges as the lack of predictive biomarkers for identification of subgroups that will benefit from novel treatment strategies.

First draft submitted: 9 February 2020; Accepted for publication: 22 April 2020; Published online: 28 May 2020

Keywords: combination therapies • hepatocellular carcinoma • immunotherapy • tyrosine kinase inhibitor • VEGFR2

Hepatocellular carcinoma (HCC) represents the fifth most common cancer worldwide with its incidence also continuously increasing in Europe or North America [1]. In the majority of cases, HCC arises in cirrhotic livers. Despite the recommendation of regular ultrasound surveillance in cirrhotic patients, potentially leading to earlier diagnoses, many patients present with an intermediate or advanced stage of the disease, according to the Barcelona Clinic of Liver Cancer (BCLC) staging system, at the time of diagnosis [2]. The BCLC classification subdivides patients with respect to their tumor burden, the degree of liver dysfunction and performance status into five different classes (BCLC 0 and A-D). Patients with advanced disease stages, as well as patients in intermediate stages which has progressed under locoablative therapies, should receive systemic treatments as long as liver function and performance status are well preserved.

Until recently, patients with a need for systemic treatment were left to toxic and only moderately effective therapies with tyrosine kinase inhibitors (TKIs) leading to often dismal prognoses [3]. After many negative trials, novel treatments are urgently needed [4]. In this context, immunotherapies have been introduced into therapeutic algorithms of many cancers including malignant melanoma, lung cancer or hematological malignancies [5]. In patients with hepatocellular cancer, such therapies seemed particularly promising, since almost all HCC develop in cirrhotic livers that bear an immunosuppressive environment that might be modulated by, for example, checkpoint inhibitors [6]. In line, early Phase I/II trials raised hope for a swift introduction into therapeutic algorithms for patients with HCC [7,8]. Moreover, ramucirumab, a novel antibody directed against VEGFR2 has demonstrated efficacy when used in patients with elevated serum AFP levels [9]. Thus, in contrast to the period before 2016, plenty of different compounds are now available for use in HCC (Table 1). However, as the efficacy of single agents used sequentially still appears limited, combination therapies including different substance classes have been tested, which will potentially change treatment algorithms in the near future (Table 2). In this review, we summarize results

Future Medicine 
Table 1. Selected Phase III studies in the context of the hepatocellular carcinoma.

\begin{tabular}{|c|c|c|c|c|c|c|c|}
\hline Study name & NCT & Line of treatment & Experimental arm & Control arm & Primary end point & $\begin{array}{l}\text { Overall survival } \\
\text { (months) }\end{array}$ & Hazard ratio \\
\hline SHARP & NCT00105443 & 1. line & Sorafenib & Placebo & os & 10.7 vs 7.9 & 0.69 \\
\hline REFLECT & NCT01761266 & 1. line & Lenvatinib & Sorafenib & OS (noninferiority) & 13.6 vs 12.3 & 0.92 \\
\hline Imbrave 150 & NCT03434379 & 1. line & $\begin{array}{l}\text { Bevacizumab + } \\
\text { atezolizumab }\end{array}$ & Sorafenib & OS/PFS & $\mathrm{N} / \mathrm{A}^{\dagger}$ & 0.58 \\
\hline CheckMate 459 & NCT02576509 & 1. line & Nivolumab & Sorafenib & os & 16.4 vs 14.7 & $0.85(p=0.07)$ \\
\hline RESORCE & NCT01774344 & 2. line & Regorafenib & Placebo & os & 10.7 vs 7.8 & 0.63 \\
\hline CELESTIAL & NCT01908426 & 2. line and later & Cabozantinib & Placebo & os & 10.2 vs 8.0 & 0.76 \\
\hline REACH-2 & NCT02435433 & 2. line & Ramucirumab & Placebo & os & 8.5 vs 7.3 & 0.71 \\
\hline Keynote-240 & NCT02702401 & 2. line & Pembrolizumab & Placebo & os & 13.9 vs 10.6 & $0.78 p=0.02 ;$ n.s. \\
\hline
\end{tabular}

\begin{tabular}{|c|c|c|c|}
\hline Trial name/identifier & Setting & Treatment & Primary end points \\
\hline \multicolumn{4}{|l|}{ Phase $\mathrm{I} / \mathrm{II}$ trials } \\
\hline GO30140/NCT02715531† & Advanced HCC/first-line & Bevacizumab + atezolizumab & Safety, ORR, PFS \\
\hline NCT03006926 & Advanced HCC/first-line & Lenvatinib + pembrolizumab & $\begin{array}{l}\text { Dose escalation: safety, DLTs } \\
\text { Dose expansion: ORR, DOR }\end{array}$ \\
\hline NCT03418922 & Advanced HCC/first-line & Lenvatinib + nivolumab & $\begin{array}{l}\text { Part 1: DLTs, safety } \\
\text { Part 2: safety }\end{array}$ \\
\hline NCT03895970 & $\begin{array}{l}\text { Advanced hepatobiliary } \\
\text { tumors/second-line }\end{array}$ & Lenvatinib + pembrolizumab & ORR, DCR, PFS \\
\hline CheckMate 040/NCT01658878 ${ }^{\dagger}$ & Advanced HCC/first- or second-line & $\begin{array}{l}\text { Cabozantinib }+ \text { nivolumab } \pm \text { ipili- } \\
\text { mumab }\end{array}$ & Safety, ORR \\
\hline COSMIC-021/NCT03170960 & Advanced solid tumors, $\mathrm{HCC}$ /first-line & Cabozantizib + atezolizumab & $\begin{array}{l}\text { Dose escalation: MTD, recommended dose } \\
\text { Dose expansion: ORR }\end{array}$ \\
\hline CaboNivo/NCT03299946 & Locally advanced HCC/neoadjuvant & Cabozantizib + nivolumab & $\begin{array}{l}\text { Safety, number of patients who complete } \\
\text { preoperative treatment and proceed to } \\
\text { surgery }\end{array}$ \\
\hline CAMILLA/NCT03539822 & Advanced GI tumors, $\mathrm{HCC} /$ second-line & Cabozantizib + durvalumab & MTD \\
\hline NCT03347292 & Advanced HCC/first-line & Regorafenib + pembrolizumab & Safety, DLTs \\
\hline REGOMUNE/NCT03475953 & Advanced GI tumors, $\mathrm{HCC} /$ second-line & Regorafenib + avelumab & $\begin{array}{l}\text { Part 1: recommended Phase II dose of } \\
\text { regorafenib } \\
\text { Part 2: ORR }\end{array}$ \\
\hline NCT02572687 & $\begin{array}{l}\text { Advanced solid tumors, } \\
\mathrm{HCC} / \text { second-line and AFP } \geq 1.5 \times \text { upper } \\
\text { limit of normal }\end{array}$ & Ramucirumab + durvalumab & DLTs \\
\hline NCT02082210 & $\begin{array}{l}\text { Advanced solid tumors, } \\
\mathrm{HCC} / \text { second-line }\end{array}$ & Ramucirumab + emibetuzumab & $\begin{array}{l}\text { Part A: DLTs } \\
\text { Part B: ORR }\end{array}$ \\
\hline NCT02423343 & $\begin{array}{l}\text { Advanced solid tumors, } \\
\mathrm{HCC} / \text { second-line and AFP } \geq 200 \mathrm{ng} / \mathrm{ml}\end{array}$ & Galunisertib + nivolumab & Phase Ib: MTD \\
\hline \multicolumn{4}{|l|}{ Phase III trials } \\
\hline LEAP-002/NCT03713593 & Advanced HCC/first-line & $\begin{array}{l}\text { Lenvatinib + pembrolizumab vs } \\
\text { lenvatinib + placebo }\end{array}$ & PFS, OS \\
\hline COSMIC-312/NCT03755791 & Advanced HCC/first-line & $\begin{array}{l}\text { Cabozantinib + atezolizumab vs } \\
\text { sorafenib vs cabozantinib }\end{array}$ & PFS, OS \\
\hline \multicolumn{4}{|c|}{$\begin{array}{l}\text { †Trials include other cohorts. } \\
\text { DCR: Disease control rate; DLT: Dose-limiting toxicity; DOR: Duration of response; GI: Gastrointestinal; HCC: Hepatocellular carcinoma; MTD: Maximum tolerated dose; ORR: Objective } \\
\text { response rate; OS: Overall survival; PFS: Progression-free survival. } \\
\text { Modified with permission from [10]. }\end{array}$} \\
\hline
\end{tabular}

from recent clinical trials analyzing these novel substances in the context of HCC. Furthermore, we discuss current indications and outline a novel treatment algorithm for use in HCC patients in need for systemic therapy. 


\section{First-line systemic therapy}

\section{Tyrosine kinase inhibitors}

Systemic therapies are recommended for patients in advanced disease stages (BCLC stage C) or for patients in intermediate-stage disease stages (BCLC stage B) that are not (or no longer) eligible for locoregional therapies [3]. In 2008, the Sorafenib Hepatocellular Carcinoma Assessment Randomized Protocol (SHARP) trial established sorafenib as the standard for systemic treatment of HCC [11]. Sorafenib is a TKI, targeting mainly VEGFR2, PDGFR and KIT [12]. The SHARP study analyzed the efficacy and safety of sorafenib compared with placebo for first-line therapy of HCC patients with well-preserved liver function (Child-Pugh A), that were not eligible for surgical or locoregional therapies. Sorafenib was associated with a significantly increased median survival of 10.7 months compared with 7.9 months in patients receiving placebo (hazard ratio [HR]: 0.69; $\mathrm{p}<0.001$ ). In line, progression-free survival (PFS) was significantly longer with sorafenib (5.5 vs 2.8 months; $p<0.001)$, response rates were unaffected by the treatment ( $2 \%$ with sorafenib; $1 \%$ with placebo [11]). Similar efficacy was reported from Asia-Pacific trial (overall survival [OS]: 6.5 vs 4.2 months; HR: 0.68; $p=0.014$ [13]). Both studies demonstrated an unfavorable toxicity profile with diarrhea, hand-foot syndrome, fatigue and anorexia representing the most common side effects. In a recently published meta-analysis sorafenib was demonstrated to be particularly efficient in those patients without extrahepatic spread, with hepatitis $\mathrm{C}$ virus infection and low neutrophil-to-lymphocyte ratio [14].

Since the introduction of sorafenib many other compounds including erlotinib [15], brivanib [16,17], sunitinib [18], linifanib [19] and everolimus [20] have been tested in first-line treatment of HCC, without showing superiority (or at least noninferiority) to sorafenib (summarized in [21]). Therefore, sorafenib remained the sole first-line option until lenvatinib, another TKI, was shown as noninferior in 2018 [22].

Similar to sorafenib, lenvatinib represents an orally available TKI, targeting VEGFR1-3, FGFR1-4, PDGFR, RET and KIT [23]. In the context of HCC, lenvatinib was tested within the REFLECT-trial, representing an open-label, multicenter, noninferiority trial comparing sorafenib with lenvatinib in previously untreated patients with histologically proven advanced (or intermediate but not eligible for transarterial chemoembolization [TACE]) HCC. Although the study showed only a slight (and not significant) improvement in median survival in the lenvatinib arm (13.6 vs 12.3 months), there was a significant improvement in tumor response (objective response rate [ORR]: 24.1 vs $9.2 \% ; \mathrm{p}=0.001$ ) and time to progression (8.9 vs 3.7 months; $\mathrm{p}<0.0001$ ). Response rates were significantly higher in patients receiving lenvatinib (18.8 vs 6.5\%; $\mathrm{p}<0.001$ according to Response Evaluation Criteria In Solid Tumors [RECIST] 1.1), arguing for a use of lenvatinib when tumor shrinkage is warranted. Further subgroup analyses showed that Asian patients, patients with hepatitis B virus infection and patients with high AFP serum concentrations ( $>200 \mathrm{ng} / \mathrm{ml}$ ) demonstrated a particular benefit from treatment with lenvatinib. Lenvatinib was associated with more frequent side effects than sorafenib, which might be due to longer treatment duration in the lenvatinib arm. Important high-grade adverse events were hypertension and weight loss for lenvatinib, as well as skin toxicity and diarrhea for sorafenib, respectively. Based on these data, current guidelines recommend both sorafenib and lenvatib for frontline treatment of unresectable HCC that are not amendable to surgery or transarterial therapies in case of BCLC B [24].

\section{Immunotherapy}

Tumor-associated antigens bound to major histocompatibility complex molecules are located on the surface of immune-presenting cells. Within lymph nodes, these cells present such antigens to immature $\mathrm{T}$ cells, which become activated and develop into so-called CD8-positive activated $\mathrm{T}$ cells that are able to recognize antigens located on tumor cells and upon activation, induce tumor cell death. This process is negatively regulated by so-called checkpoints such as PD-1 protein and CTLA4. PD-1 is a receptor, expressed on activated T and B cells as well as on myeloid cells. Activation of PD-1 by its ligands PD-L1 and PD-L2 inhibits T-cell activity, thereby causing immunosuppression. While PD-L1 is expressed in most tissues, PD-L2 is only expressed by antigenpresenting cells, highlighting the much more prominent function of PD-L1 in cancer immunity. Considering the immunosuppressive activity of the PD-1/PD-L1 complex, preventing the activation of the PD-1 receptor might help to restore the ability of immune cells to recognize and kill tumor cells. In contrast to PD-1/PDL1, CTLA4, another immune checkpoint protein, which is mainly expressed on $\mathrm{T}$ cells, is most abundant in lymph nodes and specifically regulates the proliferation of activated lymphocytes. While in a physiological setting, CTLA4 is mainly responsible for terminating T-cell activity and for preventing an excess in T-cell responses, under malignant conditions, it inhibits the activation, proliferation and production of tumor antigen-activated $\mathrm{T}$ 
cells in the tumor microenvironment. Like anti-PD-1/PD-L1-directed antibodies, anti-CTLA-directed treatments may redirect the immune system to attack the tumor. Antibodies against both PD-1/PD-L1 and CTLA4 have proven efficacy in manifold tumor entities [25]. Most HCC arise in an immunosuppressive microenvironment that might be 're-activated' by immunomodulatory treatments [6]. Various immunotherapies have been tested in the context of HCC. Vaccinations including peptide vaccines against glypican-3 and oncolytic and dendritic cell vaccines were analyzed in early phase clinical trials and have demonstrated promising results (summarized in [26]). Moreover, JX-594 (an oncolytic pox virus vaccine) demonstrated activity in a Phase I study including ten patients with advanced HCC [27]. Based on these data, the Phase III PHOCUS trial (NCT02562755) was initiated, but was stopped after a first interim analysis according to a recent press release [28]. Results from these and other (e.g., HEPAVAC-101, NCT03203005, NCT03071094 and MASTERKEY-318) studies will further clarify the role of vaccine immunotherapy in HCC (summarized, e.g., in [29]).

Checkpoint inhibitors were recently introduced as a new class of substances for therapy of solid tumors [30]. The principle of these inhibitors is to overcome immune tolerance against the tumor, which is mediated by certain inhibitory molecules such as PD-1, the PD-1 ligand, PDL-1 or CTLA-4. In the context of HCC, the CheckMate040 study, a nonrandomized Phase I/II study, which included 56 patients with therapy-naive patients, suggested that the checkpoint inhibitor nivolumab might be effective for first-line therapy of patients with advanced HCC (ORR of 13\% and 6- and 9-month survival rates of 89 and $82 \%$, respectively [7]). Just recently, the results of the much larger, randomized CheckMate-459 study investigating the efficacy of nivolumab in Phase III design were presented [31]. In this study, a total of 743 patients were randomized into an experimental arm receiving nivolumab (240 mg intravenously every 2 weeks [Q2W]) and a control arm receiving sorafenib. However, no difference in OS and PFS was demonstrated in either the IIT or PD-L1-positive cohort (OS IIT cohort 16.4 vs 14.7 months; $\mathrm{p}=0.0752$ and PFS IIT cohort 3.7 vs 3.8 months). Although tumor response was slightly better for nivolumab (ORR: 15 vs 7\%), disease control rates were comparable between both arms ( 55 vs $58 \%$ ). As expected, the toxicity profile of nivolumab was significantly better than that of sorafenib. Although many experts considered results of CheckMate-459 as 'clinically meaningful', the study remains statistically negative and nivolumab should not be used in routine care of patients with untreated, advanced HCC.

Besides nivolumab, the combination of bevacizumab (VEGF-antibody) and atezolizumab (PD-L1 antibody) was recently tested for first-line treatment of patients with HCC. First, the multi-arm Phase Ib GO301240 study had provided evidence for the efficacy of this combination by randomizing 119 patients with advanced HCC to receive either the combination of bevacizumab/atezolizumab or a atezolizumab as a single agent [31]. In this analysis, the doublet was associated with excellent tumor response (12\% complete remission, $24 \%$ partial remission and 35\% stable disease according to RECIST 1.1) and superior to single therapy in terms of PFS (5.6 vs 3.4 months; HR: 0.55). These results were confirmed by data from the Phase III, multicenter IMBRAVE-150 study [32]. In this study, a total of 501 patients with advanced HCC were randomized between the combination of atezolizumab plus bevacizumab in the experimental arm and sorafenib in the control arm. OS and PFS were tested in parallel as primary end points. The combination was associated with a significantly improved survival: in the combination arm 6-month survival rate was $85 \%$, compared with $72 \%$ in the sorafenib arm. Median survival was not yet achieved in the combination arm; in the sorafenib arm, it was 13.2 months (HR: 0.55). Similarly, PFS was significantly longer in patients receiving immunotherapy than in those receiving TKI (6.8 vs 4.3 months; HR: 0.59). Further subgroup analyses confirmed the superiority of the combination therapy in all subgroups. However, a somewhat weaker effect was observed in patients with nonviral hepatitis (HR: 0.91). Similar to GO301240 (and to CheckMate 459), administration of immunotherapy resulted in a remarkable response rates $(27 \mathrm{vs} 12 \%$; $<<0.0001)$. With regard to toxicity, IMBRAVE-150 did not reveal any new aspects: immunotherapy was less toxic than TKI therapy, which also translated into a better quality of life for the patients treated with atezolizumab plus bevacizumab. The authors of the study concluded that atezolizumab plus bevacizumab should be considered as a practice-changing treatment for patients with unresectable HCC, who have not received prior systemic therapy.

Further studies are currently underway to test the efficacy and safety of other combinations (Table 2). Promising data are available for the combination of lenvatinib and pembrolizumab, which is tested within an open-label, Phase Ib study including 104 HCC-patients with BCLC stage B (not amenable for TACE) or BCLC C, Child-Pugh class A. At present, data from the first 67 patients enrolled by 31 December 2018 are available [33]. Serious adverse events occurred in $62.7 \%$ of all patients, ORR was $44.8 \%$. Results thus compared favorably with the lenvatinib arm of REFLECT trial (ORR: 24.1\%). Median duration of response was 18.7 months, highlighting the tremendous potential of this combination. A Phase III trial (LEAP-002; NCT03713593) is ongoing. Moreover, just recently, 
results from the cabozantinib cohort of the CheckMate-040 study were presented. Within this cohort, patients with advanced HCC ( $\sim 50 \%$ therapy naive) were randomized between a doublet arm receiving nivolumab/cabozantinib and a triplet arm receiving nivolumab/ipilimumab/cabozantinib. In both arms, high response rates (19 and 29\%, respectively) were observed, which translated in numerically high PFS (5.4 vs 6.8 months) and OS (21.5 months vs not reached) in both arms. Toxicity was comparable to other combination therapies ( 47 vs $71 \%$ grade $3 / 4$ events [34]).

In summary, IMBRAVE-150 will likely change the way HCC is treated in the near future. Other combinations are currently being tested and it remains to be seen how available results from smaller studies will translate into large Phase III trials, and how this will compare with the current data from IMBRAVE-150. Further progress in immunotherapy for HCC will critically rely on the identification of predictive biomarkers that allow early identification of 'responders' [35]. It should be critically noted, that previous studies on immunotherapy have exclusively been conducted in patients with good liver function (Child-Pugh A). Currently, it is unclear to what extent this effect can be transferred to patients with impaired liver function.

\section{Cytotoxic chemotherapy}

HCC does not respond to classical chemotherapy. Nevertheless, recently, the EACH study [36] demonstrated increased PFS/ORR and a trend toward an improved survival in Asian HCC patients treated with FOLFOX4 compared with patients that received placebo. It was recently demonstrated that oxaliplatin induces immunogenic cell death and activates an antitumor immune response [37]. In line, a potential synergistic role of oxaliplatin with immune checkpoint blockades was suggested in an animal model for colorectal cancer [38]. Based on the these data, a Phase II trial of SHR-1210 plus FOLFOX4 was recently initiated in Chinese patients with advanced HCC (NCT03092895, summarized in [39]).

\section{Transarterial chemoembolization}

TACE is the standard palliative therapy for patients with HCC in stage BCLC B. Additional systemic chemotherapy could improve the efficacy of TACE. However, previous studies analyzing combinations of TACE and systemic therapy have all yielded negative results $[40,41]$. Recently, the TACTICS study, a prospective multicenter randomized trial to evaluate the effect of a combination of TACE + sorafenib compared with TACE alone revealed surprisingly promising results [42]. Within this study, patients were treated with $400 \mathrm{mg}$ sorafenib per day for 2-3 weeks before the first TACE, followed by $800 \mathrm{mg}$ sorafenib once daily and TACE 'on demand'. Of note, the study used an innovative end point, time to untreatable (unTACEable) progression, defined as tumor progression into 'no longer treatable by TACE', transient deterioration of liver function to Child-Pugh class $\mathrm{C}$ or occurrence of macrovascular invasion or extrahepatic infestation. Interestingly, a significant improvement of this end point was shown by the addition of sorafenib. Data for OS are not yet available. Combinations of immunotherapy and TACE are currently being evaluated (summarized in $[29,43])$.

\section{Selective internal radiation therapy}

Radioembolization techniques apply radioactive substances (most often Yttrium-90 containing microspheres) via the hepatic artery. Data from the European SARAH study and the Asian SIRVeniB study, which compared the toxicity and efficacy of selective internal radiation therapy (SIRT) with that of sorafenib failed to demonstrate superiority of SIRT over sorafenib in patients with nonsystemically pretreated HCC. However, the SIRT arm performed significantly better in terms of toxicity and quality of life $[44,45]$. In this context, the SORAMIC study analyzed efficacy and safety of the combination of SIRT plus sorafenib in patients with advanced HCC in a prospective randomized Phase II design [46]. The intent-to-treat cohort included 216 patients in the SIRT plus sorafenib arm and 208 patients in the sorafenib arm. Median OS was comparable in both arms (12.1 vs 11.4 months; HR: 1.01). Analysis of the per protocol cohort showed similar results. In further subgroup analyses, patients under 65 years of age (HR: 0.65; $\mathrm{p}=0.05$ ), patients without liver cirrhosis (HR: 0.46; $\mathrm{p}=0.02$ ) and patients with nonalcoholic etiology (HR: $0.63 ; \mathrm{p}=0.012$ ) seemed to benefit particularly from the combination. The combination therapy was associated with slightly increased toxicity (grade $\geq 3$ side effects in $64.8 \%$ of patients in the combination arm vs $53.8 \%$ in the patients receiving sorafenib). Overall, none of the available studies support the use of SIRT in (unselected) patients with advanced HCC eligible for systemic therapy. 


\section{Second/later-line therapy}

Tyrosine kinase inhibitors

In the SHARP/ASIAN and REFLECT studies, it was shown that administration of TKIs only leads to relatively short periods of tumor control. Based on data from the RESORCE- and CELESTIAL-studies, both regorafenib and cabozantinib are approved for use in patient's refractory to sorafenib $[47,48]$. RESORCE compared the efficacy of regorafenib in patients after failure of a sorafenib-based first-line therapy against placebo [47]. The primary end point was OS; secondary end points were PFS, ORR and safety profile. The study was positive for its primary end point (10.6 months in the regorafenib group vs 7.8 months, HR: 0.63$)$. Moreover, regorafenib significantly prolonged time to disease progression (3.1 vs 1.5 months). The efficacy of the substance was consistent in all relevant subgroups. Sequential administration of sorafenib and regorafenib resulted in an OS of 26 months compared with 19 months in patients receiving only sorafenib as first-line and placebo as second-line treatment [49]. Cabozantinib is another TKI targeting VEGFR1-3, MET and AXL [50]. The CELESTIAL study compared the efficacy of cabozantinib in patients after failure of a sorafenib-based first-line therapy against placebo [48]. Cabozantinib led to a significant improvement in OS (10.2 months in the cabozantinib arm vs 8.0 months in the placebo arm) with acceptable toxicity. Other oncological end points (PFS; ORR) were also positively influenced. As already pointed out, the combination of cabozantinib and checkpoint inhibitors was recently tested within the cabozantinib cohort (cohort 6) of the CheckMate-040 trial. This arm featured both sorafenib-naive and sorafenib-experienced patients. A separate evaluation for these two groups is not yet available; nevertheless, the combination was associated with numerically high ORR, PFS and OS rates (see above [34]).

Since RESORCE and CELESTIAL did not include an active comparative arm, it is unclear whether one substance is superior or inferior to the other. Biomarkers that enable the selection of patients for one of the substances have not yet been identified. In this context, analyses of blood samples from the RESORCE study recently identified a total of five proteins (angiopoietin 1, cystatin B, the latency-associated peptide of TGF- $\beta 1$, oxidized low-density lipoprotein receptor 1 and $\mathrm{C}-\mathrm{C}$ motif chemokine ligand 3) that were associated with prolonged survival upon treatment with regorafenib [51]. In addition, nine plasma miRNA (MIR30A, MIR122, MIR125B, MIR200A, MIR374B, MIR15B, MIR107, MIR320 and MIR645) were correlated with an improved survival. To what extent these findings will become clinically relevant remains to be seen.

\section{Ramucirumab}

In 2015, the REACH study demonstrated that, after the failure of sorafenib, HCC patients with high AFP serum concentrations $(>400 \mathrm{ng} / \mathrm{ml}$ ) may benefit from therapy with the VEGFR2 antibody ramucirumab (7.8 months for ramucirumab vs 4.2 months for placebo; $\mathrm{p}=0.006$; [52]). This retrospective analysis led to the REACH-2 study, which investigated the efficacy of ramucirumab in the second-line treatment of HCC specifically in patients with baseline AFP $>400 \mathrm{ng} / \mathrm{ml}$ in a randomized Phase III design [52]. In this study, patients with advanced HCC and baseline AFP $>400 \mathrm{ng} / \mathrm{ml}$ who were progressive under first-line sorafenib therapy were randomized to an arm receiving the VEGFR2 antibody ramucirumab or placebo. The administration of ramucirumab resulted in a small but significant improvement in survival ( 8.5 months in the ramucirumab arm vs 7.3 months in the placebo arm) with an acceptable toxicity profile. Importantly, the study confirms the feasibility of biomarker-guided therapies in HCC.

\section{Immunotherapy}

After the failure of sorafenib, both pembrolizumab (an anti-PD-1 monoclonal antibody) and nivolumab (a fully humanized monoclonal antibody against PD-1) demonstrated efficacy in Phase Ib studies (CheckMate-040 [7] and Keynote-224 [8]). However, for pembrolizumab, these results could not be confirmed in the Phase III, randomized double-blind keynote-240 trial, which included a total of 413 patients with pretreated advanced HCC (31790344) at 119 medical centers in 27 countries. The patients received either pembrolizumab or placebo. The median OS was 13.9 months in the pembrolizumab arm versus 10.6 months in the placebo arm (HR: $0.781 ; \mathrm{p}=0.0238$ ), the median PFS was 3.0 versus 2.8 months (HR: 0.718; $\mathrm{p}=0.0022$ ). However, since the prespecified alpha level was significantly lower, the study must be considered statistically negative. Similar to PD-L1 antibodies, CTLA-4 antibodies were also tested in second-line therapy of HCC; Sangro et al. described promising results (response rate was $17.6 \%$ and a median time to progression was 6.48 months) from 21 patients treated with tremelimumab at a dose of $15 \mathrm{mg} / \mathrm{kg}$ intravenously every 90 days [53]. Based on the promising results of the CheckMate-040 trial, which reported an ORR of $14 \%$ and median OS of 16 months, the efficacy and safety of the combination of 


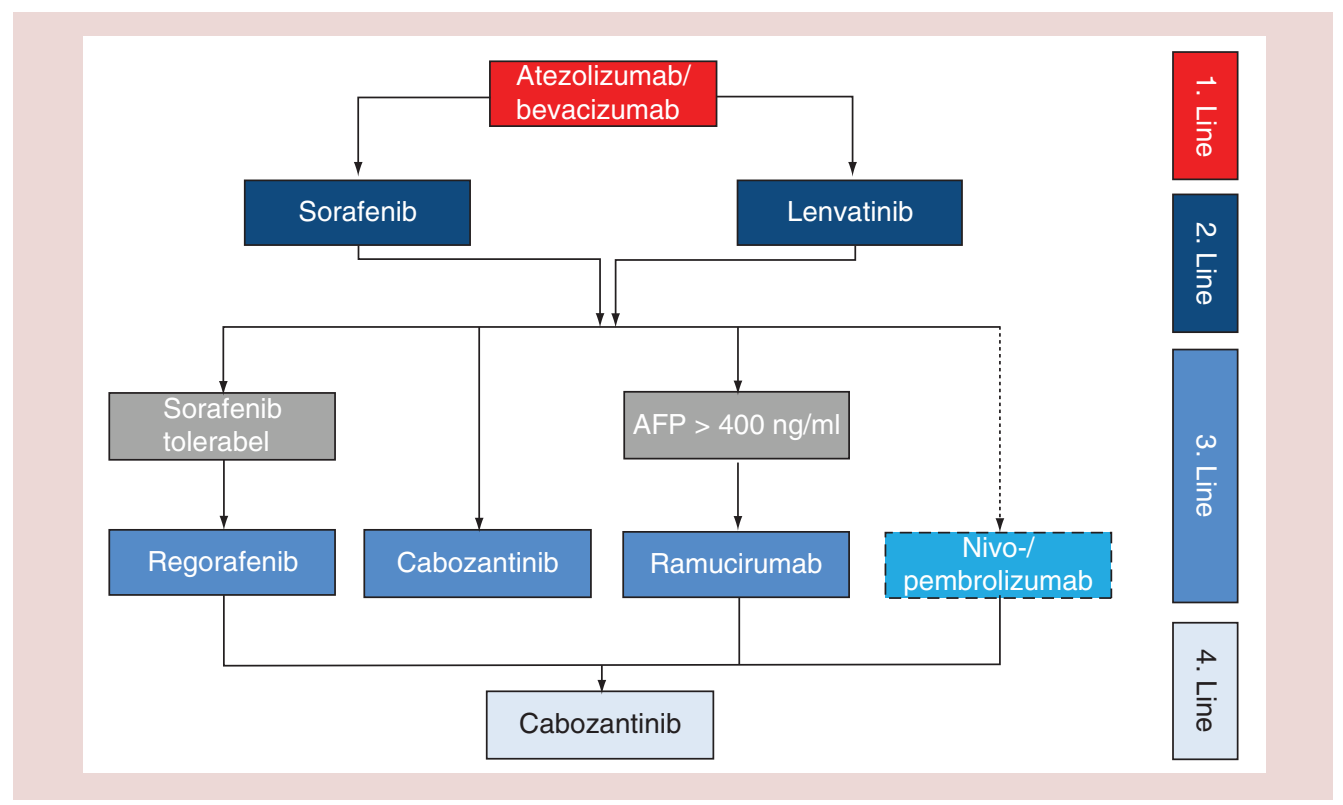

Figure 1. Proposed treatment algorithm of medical treatment in hepatocellular carcinoma patients.

nivolumab and ipilimumab was tested in sorafenib treated patients with advanced HCC [34]. One hundred and forty eight patients were randomized into three arms: nivolumab $1 \mathrm{mg} / \mathrm{kg}+$ ipilimumab $3 \mathrm{mg} / \mathrm{kg}$ every 3 weeks (Q3W) (four doses) or nivolumab $3 \mathrm{mg} / \mathrm{kg}+$ ipilimumab $1 \mathrm{mg} / \mathrm{kg} \mathrm{Q} 3 \mathrm{~W}$ (four doses), each followed by $\mathrm{n} 240 \mathrm{mg}$ Q2W, or nivolumab $3 \mathrm{mg} / \mathrm{kg}$ Q2W + ipilimumab $1 \mathrm{mg} / \mathrm{kg}$ Q6W. Overall, ORR was 31\% (with a median duration of response of 17 months), disease control rate was $49 \%$ and 24 -month survival rate was $40 \%$. Of note, the first arm demonstrated the most promising efficacy (OS: 23 months). Interestingly, the different combinations were well tolerated, potentially offering a novel treatment option for patients with pretreated HCC. The combination of nivolumab, ipilimumab and cabozantinib [34] is reported above.

\section{Conclusion}

After a decade of negative trials, various new drugs have entered the field and in some cases demonstrated superior efficacy compared with sorafenib when used as first-line treatments for patients with HCC. From the IMBRAVE150 study, it can be expected that the combination of atezolizumab plus bevacizumab will represent the new standard for untreated patients in the near future, optimal treatment sequences are only poorly investigated and remain to be defined (Figure 1). Moreover, currently, besides AFP, no biomarker is available for the identification of patients that will benefit from a certain treatment. Current data highlights that specific subgroups of patients (such as patients with nonviral etiology of liver cirrhosis) might benefit to a much lesser extend from the current advances and represent a group with a high need for novel treatments. Thus, the introduction of novel combination therapies into therapy of patients with HCC does not represent the 'end of history' but only a novel chapter on the way to an optimal treatment of all patients with primary liver cancer.

\section{Future perspective}

Recent results from large Phase III trials have somewhat overshadowed results from Phase II trials analyzing molecular guided strategies in patients with HCC. Based on data from molecular analysis on HCC samples (e.g., [54]) current studies, addressing TGF- $\beta 1-$, MET-, BRAF- and FGFR4-pathways, have generated promising results with well-tolerated active agents suitable for future combinations [10]. Such trials might open the door for personalized approaches in HCC in order to provide each individual patient with an optimal treatment. Innovative trial designs, similar to other areas of oncology, are warranted to allow the early testing of drug combinations as well as defining the optimal sequence of single and combined agents. 
Financial \& competing interests disclosure

The authors have no relevant affiliations or financial involvement with any organization or entity with a financial interest in or financial conflict with the subject matter or materials discussed in the manuscript. This includes employment, consultancies, honoraria, stock ownership or options, expert testimony, grants or patents received or pending, or royalties.

No writing assistance was utilized in the production of this manuscript.

Open access

This work is licensed under the Attribution-NonCommercial-NoDerivatives 4.0 Unported License. To view a copy of this license, visit http://creativecommons.org/licenses/by-nc-nd/4.0/

\section{References}

1. Fujiwara N, Friedman SL, Goossens N, Hoshida Y. Risk factors and prevention of hepatocellular carcinoma in the era of precision medicine. J. Hepatol. 68(3), 526-549 (2018).

2. Forner A, Reig M, Bruix J. Hepatocellular carcinoma. Lancet 391(10127), 1301-1314 (2018).

3. EASL-EORTC clinical practice guidelines: management of hepatocellular carcinoma. J. Hepatol. 56(4), 908-943 (2012).

4. Raoul JL, Gilabert M, Adhoute X, Edeline J. An in-depth review of chemical angiogenesis inhibitors for treating hepatocellular carcinoma. Expert Opin. Pharmacother. 18(14), 1467-1476 (2017).

5. Postow MA, Callahan MK, Wolchok JD. Immune checkpoint blockade in cancer therapy. J. Clin. Oncol. 33(17), 1974-1982 (2015).

6. Ringelhan M, Pfister D, O’Connor T, Pikarsky E, Heikenwalder M. The immunology of hepatocellular carcinoma. Nat. Immunol. 19(3), 222-232 (2018).

7. El-Khoueiry AB, Sangro B, Yau T et al. Nivolumab in patients with advanced hepatocellular carcinoma (CheckMate 040): an open-label, non-comparative, Phase I/II dose escalation and expansion trial. Lancet 389(10088), 2492-2502 (2017).

8. Zhu AX, Finn RS, Edeline J et al. Pembrolizumab in patients with advanced hepatocellular carcinoma previously treated with sorafenib (KEYNOTE-224): a non-randomised, open-label Phase II trial. Lancet Oncol. 19(7), 940-952 (2018).

9. Zhu AX, Kang YK, Yen CJ et al. Ramucirumab after sorafenib in patients with advanced hepatocellular carcinoma and increased alpha-fetoprotein concentrations (REACH-2): a randomised, double-blind, placebo-controlled, Phase III trial. Lancet Oncol. 20(2), 282-296 (2019).

10. Faivre S, Rimassa L, Finn RS. Molecular therapies for HCC: looking outside the box. J. Hepatol. 72(2), 342-352 (2020).

11. Llovet JM, Ricci S, Mazzaferro V et al. Sorafenib in advanced hepatocellular carcinoma. N. Engl. J. Med. 359(4), 378-390 (2008).

12. Activity of the Raf kinase inhibitor BAY 43-9006 in patients with advanced solid tumors. Clin. Colorectal Cancer 3(1), 16-18 (2003).

13. Cheng AL, Kang YK, Chen $\mathrm{Z}$ et al. Efficacy and safety of sorafenib in patients in the Asia-Pacific region with advanced hepatocellular carcinoma: a Phase III randomised, double-blind, placebo-controlled trial. Lancet Oncol. 10(1), 25-34 (2009).

14. Bruix J, Cheng AL, Meinhardt G, Nakajima K, De Sanctis Y, Llovet J. Prognostic factors and predictors of sorafenib benefit in patients with hepatocellular carcinoma: analysis of two Phase III studies. J. Hepatol. 67(5), 999-1008 (2017).

15. Zhu AX, Rosmorduc O, Evans TR et al. SEARCH: a Phase III, randomized, double-blind, placebo-controlled trial of sorafenib plus erlotinib in patients with advanced hepatocellular carcinoma. J. Clin. Oncol. 33(6), 559-566 (2015).

16. Johnson PJ, Qin S, Park JW et al. Brivanib versus sorafenib as first-line therapy in patients with unresectable, advanced hepatocellular carcinoma: results from the randomized Phase III BRISK-FL study. J. Clin. Oncol. 31(28), 3517-3524 (2013).

17. Llovet JM, Decaens T, Raoul JL et al. Brivanib in patients with advanced hepatocellular carcinoma who were intolerant to sorafenib or for whom sorafenib failed: results from the randomized Phase III BRISK-PS study. J. Clin. Oncol. 31(28), 3509-3516 (2013).

18. Cheng AL, Kang YK, Lin DY et al. Sunitinib versus sorafenib in advanced hepatocellular cancer: results of a randomized Phase III trial. J. Clin. Oncol. 31(32), 4067-4075 (2013)

19. Cainap C, Qin S, Huang WT et al. Linifanib versus sorafenib in patients with advanced hepatocellular carcinoma: results of a randomized Phase III trial. J. Clin. Oncol. 33(2), 172-179 (2015).

20. Zhu AX, Kudo M, Assenat $\mathrm{E}$ et al. Effect of everolimus on survival in advanced hepatocellular carcinoma after failure of sorafenib: the EVOLVE-1 randomized clinical trial. JAMA 312(1), 57-67 (2014).

21. Bruix J, Reig M, Sherman M. Evidence-based diagnosis, staging and treatment of patients with hepatocellular carcinoma. Gastroenterology 150(4), 835-853 (2016).

22. Kudo M, Finn RS, Qin S et al. Lenvatinib versus sorafenib in first-line treatment of patients with unresectable hepatocellular carcinoma: a randomised Phase III non-inferiority trial. Lancet 391(10126), 1163-1173 (2018).

23. Kudo M. Lenvatinib in advanced hepatocellular carcinoma. Liver Cancer 6(4), 253-263 (2017).

24. EASL clinical practice guidelines: management of hepatocellular carcinoma. J. Hepatol. 69(1), 182-236 (2018).

25. Kudo M. Immuno-oncology therapy for hepatocellular carcinoma: current status and ongoing trials. Liver Cancer 8(4), 221-238 (2019). 
26. Mansier O, Prouzet-Mauleon V, Jegou G et al. The expression of myeloproliferative neoplasm-associated calreticulin variants depends on the functionality of ER-associated degradation. Cancers 11(12), (2019).

27. Park BH, Hwang T, Liu TC et al. Use of a targeted oncolytic poxvirus, JX-594, in patients with refractory primary or metastatic liver cancer: a Phase I trial. Lancet Oncol. 9(6), 533-542 (2008).

28. Genetic Engineering \& Biotechnology News. Pexa-vec/nexavar combination fails Phase III trial in liver cancer. (2019). https://www.genengnews.com/news/pexa-vec-nexavar-combination-fails-phase-iii-trial-in-liver-cancer/

29. Lorenzen S, Lordick F, Loosen SH et al. Current status of immunotherapy in gastrointestinal malignancies. Z. Gastroenterol. 4(10), 1071-8322 (2020).

30. Chen L, Han X. Anti-PD-1/PD-L1 therapy of human cancer: past, present and future. J. Clin. Invest. 125(9), 3384-3391 (2015).

31. Xu J, Li J, Bai C et al. Surufatinib in advanced well-differentiated neuroendocrine tumors: a multicenter, single-arm, open-label, Phase Ib/II trial. Clin. Cancer Res. 25(12), 3486-3494 (2019).

32. Cheng A-L, Qin S, Ikeda M et al. IMbrave150: efficacy and safety results from a Phase III study evaluating atezolizumab (atezo) and bevacizumab (bev) vs sorafenib (sor) as first treatment (tx) for patients (pts) with unresectable hepatocellular carcinoma (HCC). Ann. Oncol. 30, ix186-ix187 (2019).

33. Ocean A, Noel M, Wang-Gillam A et al. Phase II monotherapy efficacy of cancer metabolism targeting SM- 88 in heavily pre-treated PDAC patients. Presented at: European Society for Medical Oncology (ESMO) Congress. Barcelona, Spain (2019).

34. Yau T, Zagonel V, Santoro A et al. Nivolumab (NIVO) + ipilimumab (IPI) + cabozantinib (CABO) combination therapy in patients (pts) with advanced hepatocellular carcinoma (aHCC): results from CheckMate 040. Presented at: GI Cancers Symposium. San Francisco, CA, USA (2020).

35. Villanueva A. Hepatocellular carcinoma. N. Engl. J. Med. 380(15), 1450-1462 (2019).

36. Qin S, Bai Y, Lim HY et al. Randomized, multicenter, open-label study of oxaliplatin plus fluorouracil/leucovorin versus doxorubicin as palliative chemotherapy in patients with advanced hepatocellular carcinoma from Asia. J. Clin. Oncol. 31(28), 3501-3508 (2013).

37. Garg AD, Dudek-Peric AM, Romano E, Agostinis P. Immunogenic cell death. Int. J. Dev. Biol. 59(1-3), 131-140 (2015).

38. Wang W, Wu L, Zhang J, Wu H, Han E, Guo Q. Chemoimmunotherapy by combining oxaliplatin with immune checkpoint blockades reduced tumor burden in colorectal cancer animal model. Biochem. Biophys. Res. Commun. 487(1), 1-7 (2017).

39. Liu X, Qin S. Immune checkpoint inhibitors in hepatocellular carcinoma: opportunities and challenges. Oncologist 24(Suppl. 1), S3-S10 (2019).

40. Tim M, Richard F, Yuk TM et al. Sorafenib in combination with transarterial chemoembolisation in patients with unresectable hepatocellular carcinoma (TACE 2): a randomised placebo-controlled, double-blind, Phase 3 trial. Lancet Gastroenterol. Hepatol. 2(8), 565-575 (2017).

41. Park JW, Kim YJ, Kim DY et al. Sorafenib with or without concurrent transarterial chemoembolization in patients with advanced hepatocellular carcinoma: the Phase III STAH trial. J. Hepatol. 70(4), 684-691 (2019).

42. Kudo M, Ueshima $\mathrm{K}$, Ikeda $\mathrm{M}$ et al. Randomised, multicentre prospective trial of transarterial chemoembolisation (TACE) plus sorafenib as compared with TACE alone in patients with hepatocellular carcinoma: TACTICS trial. Gut 4(318934), 2019-318934 (2019).

43. Singh P, Toom S, Avula A, Kumar V, Rahma OE. The immune modulation effect of locoregional therapies and its potential synergy with immunotherapy in hepatocellular carcinoma. J. Hepatocell. Carcinoma 7, 11-17 (2020).

44. Chow PKH, Gandhi M, Tan SB et al. SIRveNIB: selective internal radiation therapy versus sorafenib in Asia-Pacific patients with hepatocellular carcinoma. J. Clin. Oncol. 36(19), 1913-1921 (2018).

45. Vilgrain V, Pereira $\mathrm{H}$, Assenat $\mathrm{E}$ et al. Efficacy and safety of selective internal radiotherapy with yttrium-90 resin microspheres compared with sorafenib in locally advanced and inoperable hepatocellular carcinoma (SARAH): an open-label randomised controlled Phase III trial. Lancet Oncol. 18(12), 1624-1636 (2017).

46. Ricke J, Klumpen $\mathrm{HJ}$, Amthauer $\mathrm{H}$ et al. Impact of combined selective internal radiation therapy and sorafenib on survival in advanced hepatocellular carcinoma. J. Hepatol. 71(6), 1164-1174 (2019).

47. Bruix J, Qin S, Merle P et al. Regorafenib for patients with hepatocellular carcinoma who progressed on sorafenib treatment (RESORCE): a randomised, double-blind, placebo-controlled, Phase III trial. Lancet 389(10064), 56-66 (2017).

48. Abou-Alfa GK, Meyer T, Cheng AL et al. Cabozantinib in patients with advanced and progressing hepatocellular carcinoma. N. Engl. J. Med. 379(1), 54-63 (2018).

49. Finn RS, Merle P, Granito A et al. Outcomes of sequential treatment with sorafenib followed by regorafenib for HCC: additional analyses from the Phase III RESORCE trial. J. Hepatol. 69(2), 353-358 (2018).

50. Durante C, Russo D, Verrienti A, Filetti S. XL184 (cabozantinib) for medullary thyroid carcinoma. Expert Opin. Investig. Drugs 20(3), 407-413 (2011).

51. Teufel M, Seidel H, Kochert $\mathrm{K}$ et al. Biomarkers associated with response to regorafenib in patients with hepatocellular carcinoma. Gastroenterology 156(6), 1731-1741 (2019). 
52. Zhu AX, Park JO, Ryoo BY et al. Ramucirumab versus placebo as second-line treatment in patients with advanced hepatocellular carcinoma following first-line therapy with sorafenib (REACH): a randomised, double-blind, multicentre, Phase III trial. Lancet Oncol. 16(7), 859-870 (2015).

53. Sangro B, Gomez-Martin C, de la Mata M et al. A clinical trial of CTLA-4 blockade with tremelimumab in patients with hepatocellular carcinoma and chronic hepatitis C. J. Hepatol. 59(1), 81-88 (2013).

54. Cancer Genome Atlas Research Network. Comprehensive and integrative genomic characterization of hepatocellular carcinoma. Cell 169(7), 1327-1341 (2017). 